\title{
Research on Current Development Situation and the State of Supply and Demand of Medical Service Industry of China
}

\author{
Zilin Guo \\ Wuchang Shouyi University, Wuhan, 430064, China
}

Keywords: Medical service industry, medical and health institutions, State of supply and demand.

\begin{abstract}
China's medical service industry occupies a certain position in the service market in China. With the improvement of people's living standard, the demand for medical industry is increasing, which promotes the sustained supply growth of medical service resources. Starting from the current situation of China's medical industry, this paper analyzes the unbalanced medical resources of China and the differences between urban and rural areas in the development of China's medical industry. In view of the above problems, this paper puts forward the rationalization proposals to promote the distribution of medical and health service system and resources more reasonable in our country.
\end{abstract}

\section{Introduction}

Analysis on the Quality of the Medical Institutions of Different Types. The medical service industry in China can be divided into the following three groups: hospitals, primary health care institutions and professional public health institutions. The hospital contains the general hospital, Chinese medicine hospitals and specialist hospitals; the basic medical and health institutions mainly by the community health service centers and hospitals in the streets, township hospitals, village clinics and outpatient department, which is composed of four parts; professional public health agencies including the control center, maternal and child health hospital and health specialized disease prevention and supervision of disease prevention. We analyze the types of Chinese medicine industry in the Chinese statistical yearbook to obtain Figure 1. Red represents the professional public health institution, and blue represents the hospital. The development trend of primary health care institution is also shown as follows.

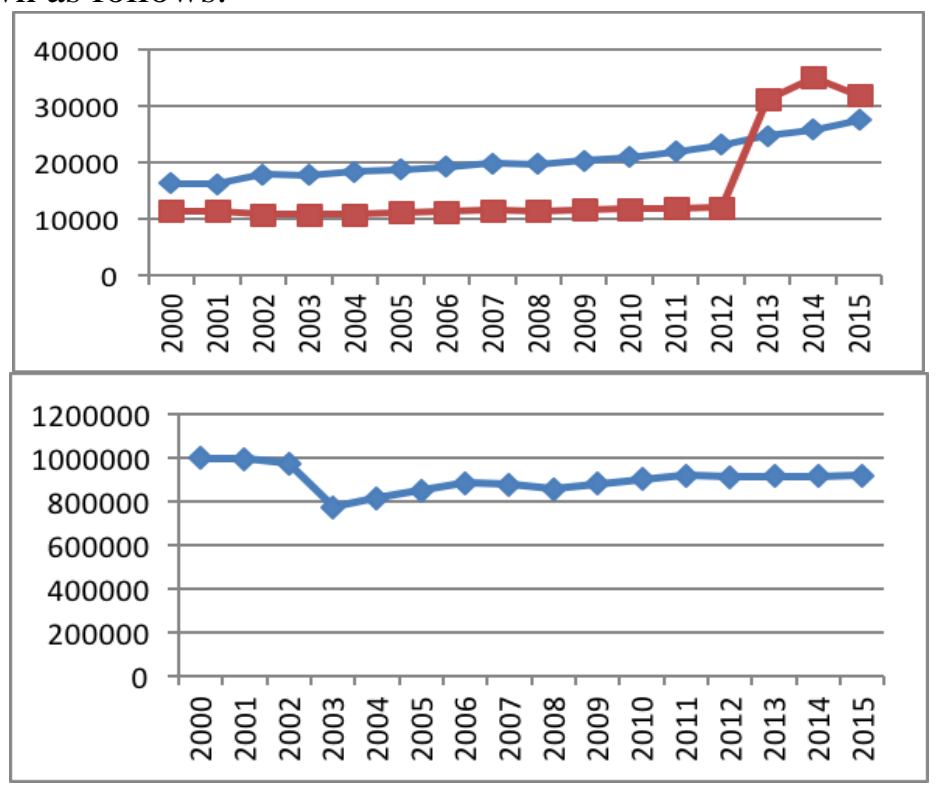

Figure 1. Development trend of medical institutions of different types in China 
From the quantitative point of view, China's medical industry in the most basic medical and health institutions, from the trend, the growth trend is relatively stable, steady growth in, further describes the establishment of basic medical institutions and rapid growth, provides great convenience for people to see doctors.

Analysis on the Quality of the Medical Personals. Medical personnel in our medical industry mainly include five groups of health technical personnel, rural doctors and health workers, other technical personnel, managerial staff and skilled workers. The number of health technical personnel in our country is shown in Figure 2. The demand for medical practitioners in our medical industry is increasing, and the medical industry is developing rapidly.

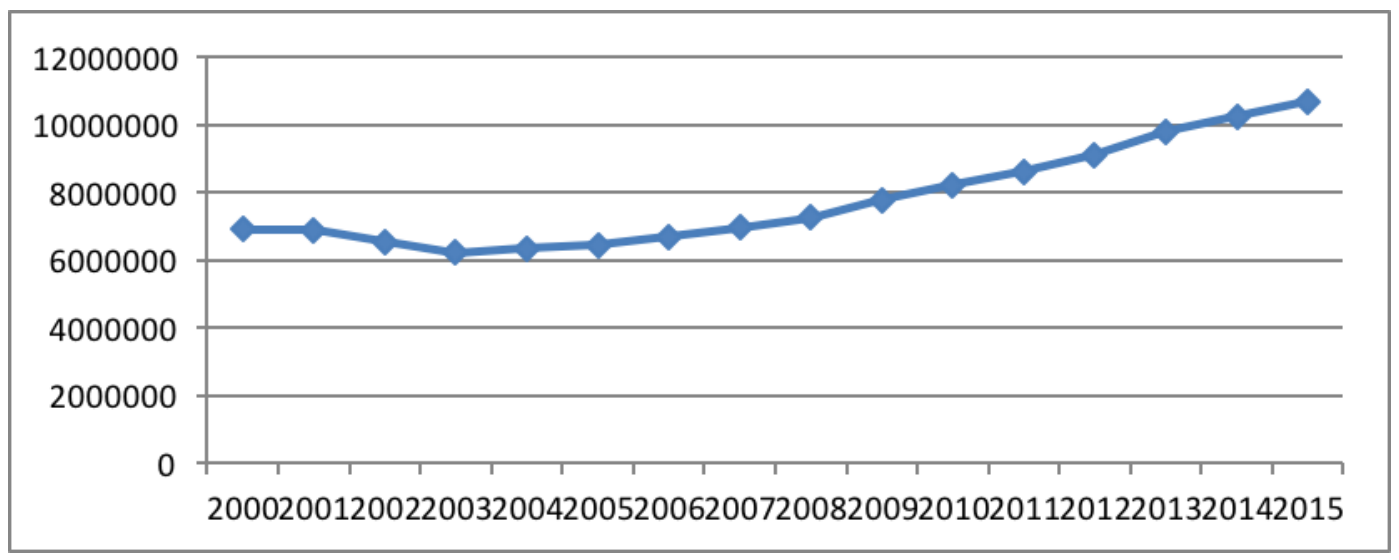

Figure 2. Quality of the medical personals of China

Analysis on the Quality of Public Hospitals and Private Hospitals. Although the public hospital is the main medical service system in China, but with the medical reform, the proportion of public hospitals will continue to decline, and guide social capital in China has entered into the medical system, thus breaking the monopoly of public hospitals, to achieve efficient allocation of resources in the fair competition. By analyzing the quantity of public hospitals and private hospitals in our country, the result of figure three is obtained. The blue represents the public hospital, and the red represents the private hospital.

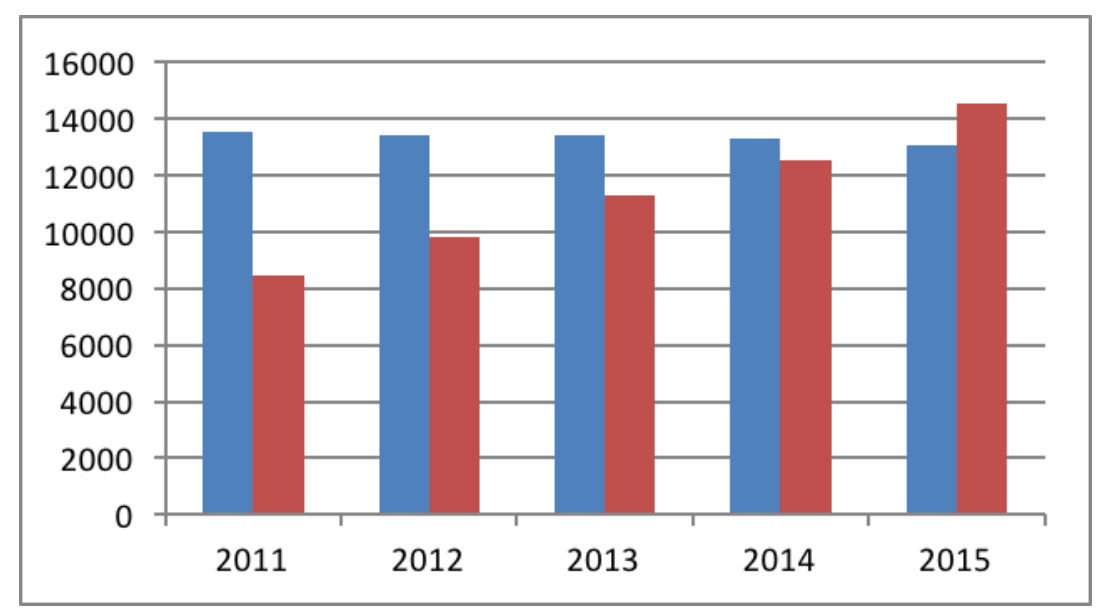

Figure 3. Quality of public hospitals and private hospitals

Through the above analysis, we can know the current situation of medical service industry in China. The number of The professional public health institutions increased significantly; the high quality medical intuitions will play an important role in China's medical market; the number of health workers is increasing, China's medical and health professionals continue to increase the demand for private hospitals; increasing the number, position in the the society will rise in the market competition, promote the rational allocation of resources; the basic medical and health institutions continue to increase due to the convenience of the people to see doctors. 


\section{State of Supply and Demand of Medical Service Industry of China}

Supply State of Medical Service Industry of China. With the continuous improvement of living standards, coupled with the aging trend intensifies, people demand for medical services increased, at the same time for the medical service quality requirements improve, the number of visits, personal health expenditure and rural health expenditure per capita in demand Chinese medical industry analysis. The analysis of the volume of outpatients is shown in the following figure.

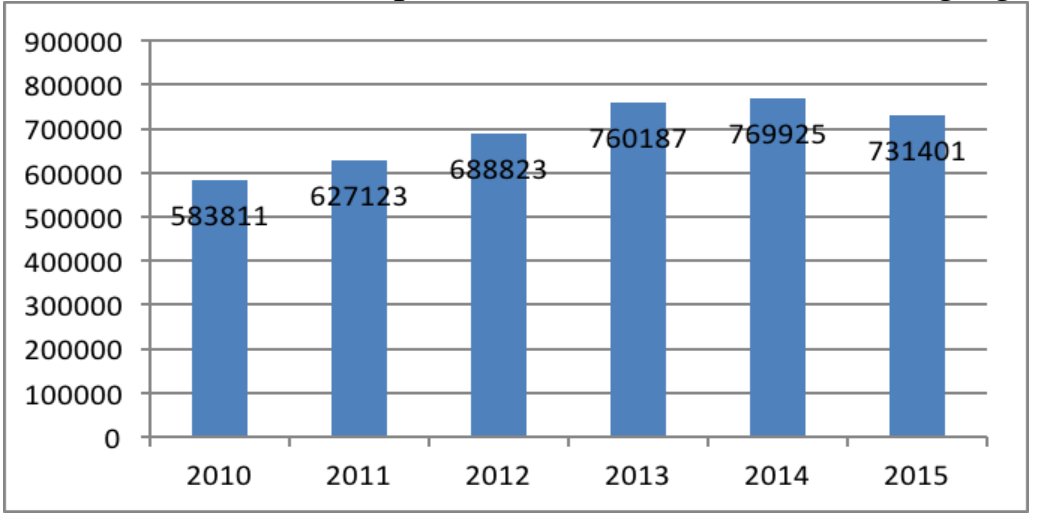

Figure 4. Trend of the volume of outpatients in China

The trend of the personal cost of health is shown in Figure 5.

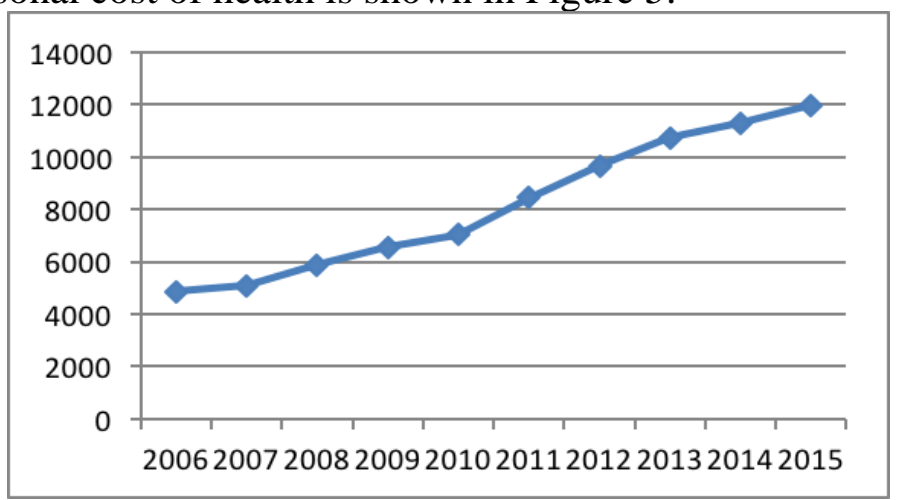

Figure 5. Trend of the personal cost of health

The average health cost of urban residents and the average health cost of rural residents are shown in Figure 6.

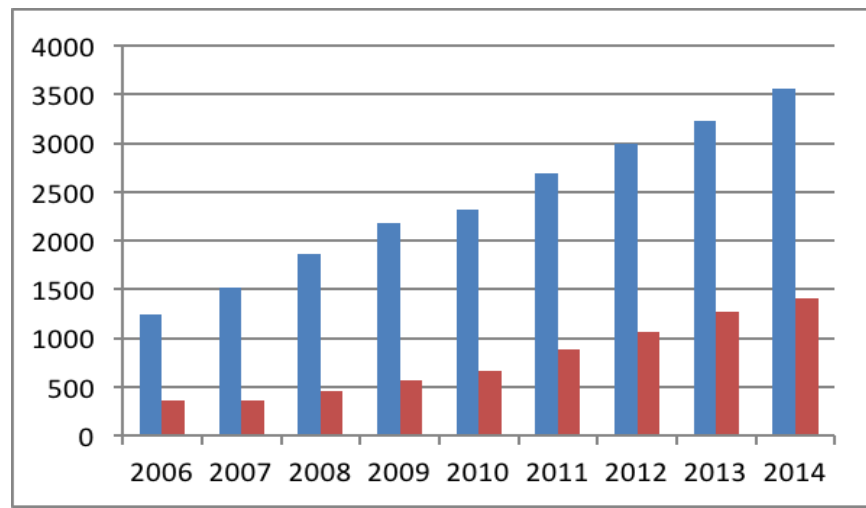

Figure 6. Average health cost of urban residents and rural residents

We can see from Figure 6, there are differences in health costs between urban and rural areas, and the differences are widening. Although the cost of health between cities and rural areas has shown an increasing trend, the trend of rural growth is far less than that of cities, which is closely related to the income gap between urban and rural areas. This also reflects the problems existing in the medical and health industry in China, and needs to be paid attention to by the government and continuously promote the balanced growth of the demand of the medical industry in china.

In short, through the above analysis, the medical service industry in China is increasing in demand. This is inseparable from the improvement of people's living standards and health importance. At the 
same time, there are also differences in the demand for medical service industry, so we need to pay more attention to it.

Demand State of Medical Service Industry of China. Facing the increasing demand for the medical service industry, our government has increased the supply of the industry to meet the needs of the people, fully play the role of the government, and better respond to changes in the market. The blue represents the health cost of government, and the red represents the health cost of society.

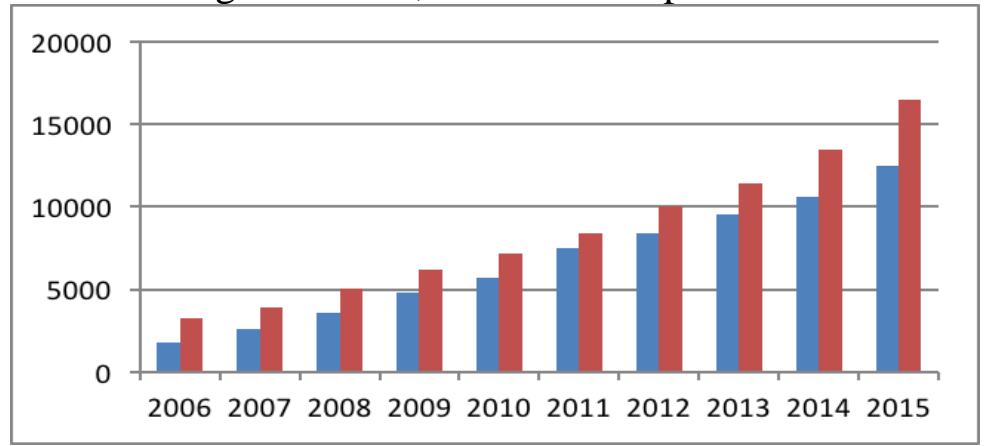

Figure 7. Health cost of government and society

The number of beds of the medical and health intuitions are shown in Figure 8.

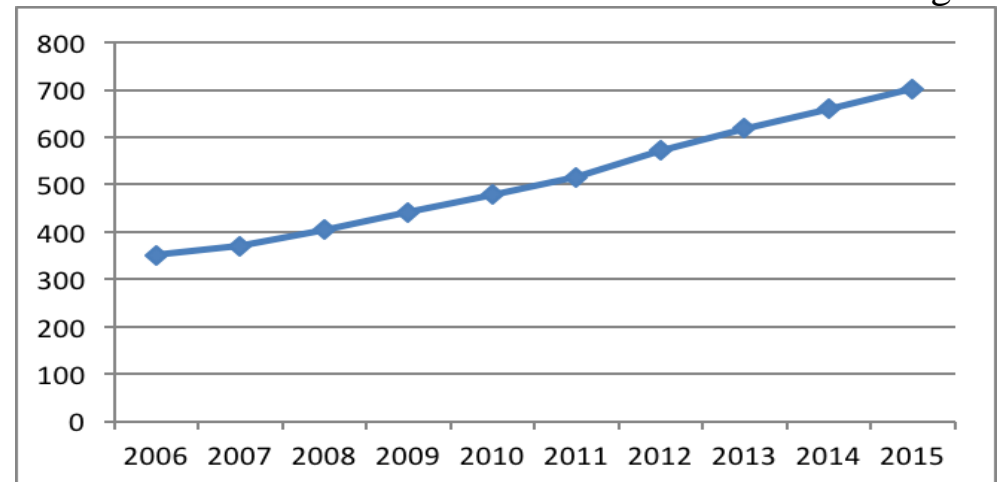

Figure 8. Number of beds of the medical and health intuitions

In conclusion, through the analysis of the supply of medical service industry in our country, our government and medical institutions for medical service supply continues to increase, we can see that China's degree of attention to the medical industry, meet growing demand, provide convenience in the aspect of supply.

Relationship of the Supply and Demand States of Medical Service Industry of China. The personal cost in health (PEH) and the government cost in health (GEH) are shown in Table 1.

Table 1. PEH and GEH from 2006 to 2015

\begin{tabular}{ccc}
\hline Year & PEH & GEH \\
\hline 2006 & 4853.56 & 1778.86 \\
2007 & 5098.66 & 2581.58 \\
2008 & 5875.86 & 3593.94 \\
2009 & 6571.16 & 4816.26 \\
2010 & 7051.29 & 5732.49 \\
2011 & 8465.28 & 7464.18 \\
2012 & 9656.32 & 8431.98 \\
2013 & 10729.34 & 9545.81 \\
2014 & 11295.41 & 10579.23 \\
2015 & 11992.65 & 12475.28 \\
\hline
\end{tabular}


Through the empirical analysis, we can see that the supply and demand of medical service industry of China affect mutually. There is a two-way causal relationship between the two variables.

\section{Conclusion}

Through the analysis of the current situation of China's medical service industry and the supply and demand situation of the market, we draw the following conclusions: high quality medical care will occupy an important position in the medical market in China. The number of private hospitals is increasing and plays an important role in the society. The primary medical and health institutions are constantly increasing, providing convenience for the common people. The demand for medical services is increasing, but the gap between urban and rural areas is too large. The supply of medical service resources has been increasing, but the total amount of medical resources is still insufficient.

\section{References}

[1] Zhang Kan, Dong Wei. A situation analysis of medical service system and policy suggestions to new health system reform in Shanghai [J]. Chinese Journal of Health Policy, 2009, 2(6): 32-35.

[2] Ma Liping, Chen Hua, Yang Tingting. Thoughts on development history of China's health service system [J]. Chinese Hospitals, 2013,17(5): 24-26.

[3] Zhang Weidong, Kong Huipang. The Empirical Research of the Influence Factors of Urban Residents Medical Demand in China [J].Chinese Health Economics, 2014,33(4): 53-54.

[4] Feng Jin, Yu Yangyang, Lou Pingyi. Medical Demand and Growing Medical Costs in ChinaBased on the Gap between Senior Citizens' Medical Costs in Urban and Rural Areas [J].Social Sciences in China, 2015(3): 85-103+207. 